\title{
Sotalol-induced torsade de pointes: management with magnesium infusion
}

\author{
Margaret A. Arstall, John T.Y. Hii, Ronald G. Lehman and John D. Horowitz
}

Cardiology Unit, The Queen Elizabeth Hospital, University of Adelaide, Woodville Road, Woodville South, Adelaide, South Australia 5011

\begin{abstract}
Summary: A 69 year old woman was treated with sotalol (320 mg daily) for intermittent atrial fibrillation. Sotalol was initially well tolerated, and reversion to sinus rhythm with sinus bradycardia occurred 4 weeks after initiation of therapy. Shortly thereafter, the patient developed recurrent syncope due to torsade de pointes. This was treated successfully with intravenous magnesium infusion and withdrawal of sotalol. Subsequently, the atrial fibrillation was adequately managed using amiodarone, with no recurrence of torsade de pointes. Development of bradycardia associated with reversion to sinus rhythm represents a potential cause of 'late' pro-arrhythmic effects of sotalol.
\end{abstract}

\section{Introduction}

Torsade de pointes (TDP) occurring during treatment with the anti-arrhythmic agent sotalol, has frequently been associated with the presence of complicating factors such as drug overdose or more commonly, hypokalaemia. ${ }^{1}$ We wish to report a case of sotalol-induced TDP in which no such complicating factor was clinically apparent.

\section{Case report}

A 69 year old woman was admitted for management of symptomatic atrial fibrillation of 5 days duration. There was a past history of mitral valvotomy. Rapid ventricular response to the atrial fibrillation persisted despite treatment with digoxin and verapamil. She had also been treated with frusemide and warfarin. Verapamil was ceased and sotalol commenced, initially at a dose of $160 \mathrm{mg}$ daily, with her other treatment unchanged. The sotalol dosage was increased to $320 \mathrm{mg}$ daily after 3 weeks. Reversion to sinus rhythm, with subsequent asymptomatic sinus bradycardia, occurred 4 weeks after initiation of sotalol treatment.

Twenty-four hours after reversion to sinus rhythm, the patient developed recurrent syncopal episodes preceded on each occasion by palpitations. Ambulatory electrocardiogram (ECG) monitoring revealed several episodes of short-lived TDP associated with pre-syncope (Figure 1). The

Correspondence: Margaret Arstall, M.B., B.S. Accepted: 23 September 1991 patient was transferred to the coronary care unit and both sotalol and digoxin were ceased. An intravenous bolus of $15 \mathrm{mmol}$ magnesium sulphate was administered over $1 \mathrm{~min}$, with no adverse effect, followed by a $24 \mathrm{~h}$ infusion at a rate of $8 \mathrm{mmol} / \mathrm{h}$. All ventricular ectopy ceased after thece bolus injection, with no recurrence of TDP.

Investigations at the time of this presentation included ECG, which revealed left bundle branch block with some incremental prolongation of the QTc ( $0.51 \mathrm{~s}$ before sotalol therapy compared to $0.56 \mathrm{~s}$ while on sotalol therapy), serum potassium level of $3.9 \mathrm{mmol} / 1 \quad(R: 3.2-5.3)$ and serum magnesium of $0.87 \mathrm{mmol} / 1(R: 0.75-1.13)$.

Therapy with amiodarone was subsequently initiated on recurrence of rapid atrial fibrillation, and the patient was discharged without recurrence of symptoms over the next 2 years. During this time the rhythm has been controlled atrial fibrillation.

\section{Discussion}

This patient developed TDP while being treated with racemic sotalol in the usual clinical dosage. The current case represents the fourth report in the literature of this phenomenon. In the three previously reported cases, ${ }^{2-4}$ no precipitating factor other than sotalol therapy could be delineated, and plasma sotalol concentrations appeared unremarkable when measured.

The issue arises as to whether this patient might have been predisposed to TDP on the basis of a congenital prolonged repolarization syndrome. ${ }^{5}$ While this remains possible, the patient had 


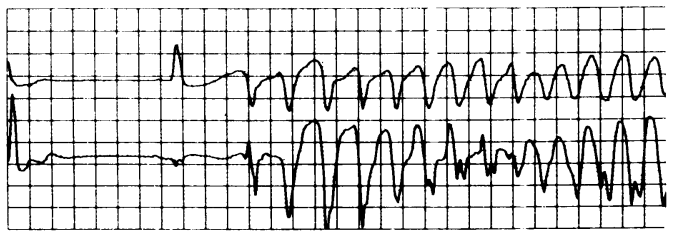

Figure 1 Ambulatory ECG monitoring of an episode of torsade de pointes.

experienced no symptoms suggestive of TDP prior to the initiation of therapy with sotalol. Furthermore, the development of TDP co-incident with reversion to sinus bradycardia per se played a part in arrhythmogenesis. It has previously been suggested that bradycardia may predispose to the development of TDP, ${ }^{6}$ but in most previously reported cases, the concurrence of TDP with sinus bradycardia might have been epiphenomenal. ${ }^{2-4.6}$ In the currently reported case, it is far more likely that reversion to sinus bradycardia, rather than gradual myocardial accumulation of sotalol, was the precipitant for TDP. Successful treatment of sotalol overdose-induced TDP with overdrive pacing also supports this view. ${ }^{6}$ Since drug-induced reversion in patients treated for atrial fibrillation may occur (as in the present case) a considerable

\section{References}

1. McKibbin, J.K., Pocock, W.A., Barlow, J.B., Millar, R.N.S \& Obel, I.W.P. Sotalol, hypokalaemia, syncope and torsade de pointes. Br Heart J 1984, 51: 157-162.

2. Krapf, R. \& Gertsch, M. Torsade de pointes induced by sotalol despite therapeutic plasma sotalol concentrations. $\mathrm{Br}$ Med J 1985, 290: 1784-1785.

3. Kuck, K.H., Kunze, K.P., Roewer, N. \& Bleifeld, W. Sotalol-induced torsade de pointes. Am Heart J 1984, 107: $179-180$

4. Sieber, C., Weiss, P. \& Follath, F. Hemiparesis in torsade de pointes under low dose sotalol therapy. Schweiz Med Worchenschr 1990, 120: 1397-1399.

5. Bauman, J.L., Bauernfeind, R.A., Hoff, J.V., Strasberg, B., Swiryn, S. \& Rosen, K.M. Torsade de pointes due to quinidine: observations in 31 patients. Am Heart J 1984, 107: $425-430$

6. Totterman, K.J., Turto, H. \& Pellinen, T. Overdrive pacing as treatment of sotalol-induced ventricular tachyarrhythmias (torsade de pointes). Acta Med Scand (Suppl) 1982, 668: $28-33$. time after initiation of therapy, this implies a possibly preferable role for early electrical reversion in such patients, to facilitate monitoring post-reversion.

This patient was treated successfully with intravenously infused magnesium, as had been done in one previously reported case of sotalol-induced $\mathrm{TDP}^{4}$ and most other cases of drug-induced TDP, irrespective of serum magnesium concentrations. Whether this patient had intracellular magnesium depletion because of longstanding loop diuretic use was not clinically apparent, but could not be excluded. The response to magnesium suggests that the aetiology of this form of TDP involves early after-depolarizations, which are suppressed by magnesium, although dispersion of refractoriness remains a possibility. ${ }^{7-9}$

Finally, the patient was managed successfully with amiodarone, which like sotalol acts fundamentally as a potassium channel blocker. ${ }^{11}$ Failure of the TDP to recur again either relates to the fact that the patient did not revert to sinus rhythm on amiodarone therapy, or reflects differences between the electrophysiological effects of these two class III anti-arrhythmic agents. ${ }^{11,12}$ Perhaps these differences are related in particular to the more frequent development of bradycardia with sotalol.

7. Tzivoni, D., Banai, S.. Schuger, C. et al. Treatment of torsade de pointes with magnesium sulphate. Circulation 1988, 77: 392-397.

8. Kaseda, S., Gilmour, R.F. \& Zipes, D.P. Depressant effect of magnesium on early afterdepolarizations and triggered activity induced by caesium, quinidine and 4-aminopyridine in canine cardiac Purkinje fibres. Am Heart J 1989, 118: $458-466$.

9. Surawicz, B. Electrophysiologic substrate of torsade de pointes: dispersion of repolarisation or early afterdepolarizations. J Am Coll Cardiol 1989, 14: 172-184.

10. Colatsky, T.J. \& Follmer, C.H. $\mathrm{K}^{+}$channel blockers and activators in cardiac arrhythmias. Cardiovasc Drug Rev 1989, 7: 199-209.

11. Mattioni, T.A., Zheutlin, T.A., Sarmiento, J.J. et al. Amiodarone in patients with previous drug mediated torsade de pointes. Am Int Med 1989, 111: 574-580.

12. Amiodarone vs Sotalol Study Group. Multicentre randomized trial of sotalol vs amiodarone for chronic malignant ventricular tachyarrhythmias. Eur Heart J 1989, 10: 685-694. 